\title{
Sorption of organophosphate esters by carbon nanotubes
}

\author{
Wei Yan ${ }^{\mathrm{a}}$, Li Yan ${ }^{\mathrm{a}}$, Jinming Duan ${ }^{\mathrm{b}}$, Chuanyong Jing a,* \\ a State Key Laboratory of Environmental Chemistry and Ecotoxicology, Research Center for Eco-Environmental Sciences, Chinese Academy of Sciences, \\ Beijing 100085, China \\ b School of Environmental and Municipal Engineering, Xi'an University of Architecture and Technology, Xi'an 710055, China
}

\section{H I G H L I G H T S}

- Oxygen-containing groups on CNTs change the sorption property for OPEs.

- Molecular configuration of OPEs has insignificant impact on their sorption.

- Hydrophobic, $\pi-\pi$ EDA and Brønsted acid-base interaction occurred between the CNTs and OPEs.

\section{A R T I C L E I N F O}

\section{Article history:}

Received 16 November 2013

Received in revised form 12 March 2014

Accepted 13 March 2014

Available online 27 March 2014

\section{Keywords:}

Organophosphate esters

Carbon nanotubes

Sorption

FTIR

Raman spectroscopy

\section{G R A P H I C A L A B S T R A C T}

The interfacial interactions between the OPE molecules and CNTs.

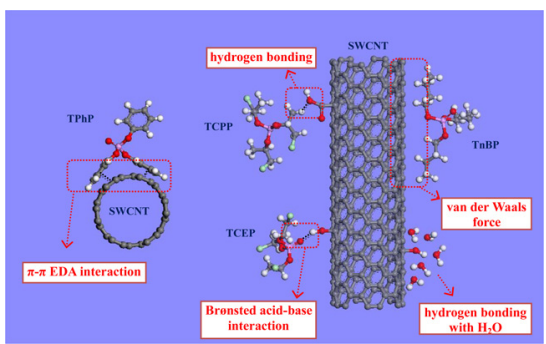

\begin{abstract}
A B S T R A C T
Insights from the molecular-level mechanism of sorption of organophosphate esters (OPEs) on carbon nanotubes (CNTs) can further our understanding of the fate and transport of OPEs in the environment. The motivation for our study was to explore the sorption process of OPEs on multi-walled CNTs (MWCNTs), single-walled CNTs (SWCNTs) and their oxidized counterparts (O-MWCNTs and O-SWCNTs), and its molecular mechanism over a wide concentration range. The sorption isotherm results revealed that the hydrophobicity of OPEs dominated their affinities on a given CNT and the $\pi-\pi$ electron donor-acceptor (EDA) interaction also played an important role in the sorption of aromatic OPEs. This $\pi-\pi$ EDA interaction, verified with Raman and FT-IR spectroscopy, could restrict the radial vibration of SWCNTs and affect the deformation vibration $\gamma(\mathrm{CH})$ bands of OPE molecules. The OPE surface coverage on CNTs, estimated using the nonlinear Dubinin-Ashtakhov model, indicated that the oxygen-containing functional groups on CNTs could interact with water molecules by H-bonding, resulting in a decrease in effective sorption sites. In addition, FTIR analysis also confirmed the occurrence of Brønsted acid-base interactions between OPEs and surface $\mathrm{OH}$ groups of SWCNTs. Our results should provide mechanistic insights into the sorption mechanism of OPE contaminants on CNTs.
\end{abstract}

(C) 2014 Elsevier B.V. All rights reserved.

\section{Introduction}

Organophosphate esters (OPEs) are a group of industrial chemicals frequently used as flame retardants and plasticizers [1]. Since the early 2000s, OPEs have been extensively used as an alternative

\footnotetext{
* Corresponding author. Tel.: +86 106284 9523; fax: +861062849523.

E-mail address: cyjing@rcees.ac.cn (C. Jing).
}

to polybrominated diphenyl ethers (PBDEs) due to the worldwide phase-out and restriction of PBDEs [2]. The global consumption of OPEs, therefore, was increased to $296000 \mathrm{t} / \mathrm{yr}$ in 2004, and all OPEs were listed as high production volume chemicals by the International Uniform Chemical Information Database [3]. A large quantity of OPEs (up to high $\mu \mathrm{g} / \mathrm{L}$ or $\mu \mathrm{g} / \mathrm{kg}$ levels) [2] could be readily released or leached into the surrounding environment, given that they are physically mixed with products rather than chemically bonded [4]. Consequently, OPEs are now frequently detected in 
various environmental matrixes, such as air [5], wastewater [6], surface water [4,7], soil [3,8], and sediment [9]. As a substitute for the banned PBDEs, however, OPEs are not as safe as initially expected. Their adverse health effects, including neurotoxicity and carcinogenic effects, have been recently confirmed [2].

To date, research efforts have primarily focused on the occurrence and toxicity of OPEs in the environment. In contrast, studies on their solid-solution interfacial behaviors, which determine the fate and transport of OPEs in the aquatic environment, are still scarce. Actually, due to the variety of substituent groups, OPEs exhibit a large variation in chemical properties, ranging from polar (e.g. trimethyl phosphate, TMP, $\log K_{\mathrm{ow}}-0.65$ [10]) to non-polar (e.g. tri(ethylhexyl) phosphate, TEHP, $\log K_{\text {ow }} 9.49$ [10]). The different hydrophobicity of OPEs results in their diverse environmental behaviors. Furthermore, the substituents play important roles in sorption processes due to electron donor-acceptor (EDA) interactions, hydrogen bonding and covalent bond formation. In addition, OPEs with different substituent groups have different molecular size and shape, which subsequently affects their sorption affinity and capacity.

Carbon nanotubes (CNTs), with an outer diameter in the nanometer range (ca. $1-100 \mathrm{~nm}$ ), comprise a class of nanoparticles with extensive $\mathrm{sp}^{2}$ carbon atoms arranged in fused benzene rings [11]. Due to their unique physicochemical properties [12], CNTs have ever-increasing industrial applications [13-15]. CNTs may contain functional groups such as hydroxyl, carboxyl, and carbonyl groups, contributing to their aggregation and sorption of organic chemicals [16]. As a consequence, the release of CNTs to the environment could greatly alter the mobility, bioavailability, and fate of organic chemicals [17].

The objective of this study was to explore the sorption behavior and mechanisms of OPEs on CNTs at the molecular level. Six OPEs with different substituent groups were used to investigate the impact of different physical-chemical properties on their sorption. Four types of CNTs, including pristine multi-walled CNTs (MWCNTs) and single-walled CNTs (SWCNTs), and their oxidized counterparts (O-MWCNTs and O-SWCNTs), were selected as sorbents to probe the specific interactions between the OPEs and CNTs with different structural and surface properties. Multiple complementary techniques including macroscopic sorption experiments, attenuated total reflectance Fourier-transform infrared (ATR-FTIR) spectroscopy, and Raman spectroscopy, were used to study the sorption mechanism. The results should further our fundamental understanding of the interactions of OPEs with carbon-based materials.

\section{Experimental}

\subsection{Sorbates and sorbents}

Six OPEs (all HPLC grade, purity >98\%) including tri(2chloroethyl) phosphate (TCEP), tri(chloropropyl) phosphate (TCPP), tributoxyethyl phosphate (TBEP), tri-n-butyl phosphate (TnBP), triphenyl phosphate (TPhP), and tri-3-cresyl phosphate (TCrP), were purchased from Dr. Ehrenstorfer GmbH (Augsburg, Germany). Selected physical-chemical properties of OPEs are listed in Table 1. The molecular structures of the six OPEs are shown in Fig. S1 in Supporting information (SI).

SWCNTs and MWCNTs were obtained from Shenzhen Nanotech Port Co., China. Prior to the sorption experiments, the carbon nanotubes were purified to remove amorphous carbon and metal catalysts using thermal and $\mathrm{NaClO}$ treatments [18]. To obtain a high level of surface oxidation, purified CNTs were treated using a $\mathrm{HNO}_{3}$ oxidation treatment previously described by Cho et al. [19]. The specific procedures of purification and oxidative treatment
Table 1

Selected physical-chemical properties of OPEs.

\begin{tabular}{llcllll}
\hline OPEs & $\mathrm{MW}^{\mathrm{a}}$ & \multicolumn{1}{c}{$C_{\mathrm{s}}{ }^{\mathrm{b}}$} & $\log K_{\mathrm{ow}^{\mathrm{c}}}$ & $\rho^{\mathrm{d}}$ & $V_{\mathrm{s}}^{\mathrm{e}}$ & $\mathrm{MV}^{\mathrm{f}}$ \\
\hline TCEP & 285.49 & 7820 & 1.44 & 1.419 & 205 & 194.85 \\
TCPP & 327.57 & 1080 & 2.59 & 1.288 & 255.61 & 243.69 \\
TnBP & 266.31 & 400 & 4 & 0.97 & 269.85 & 247.67 \\
TBEP & 398.47 & 1300 & 3.75 & 1.02 & 387.98 & 367.77 \\
TPhP & 326.28 & 1.9 & 4.59 & 1.206 & 257.86 & 297.01 \\
TCrP & 368.36 & 0.36 & 5.11 & 1.16 & 306.69 & 345.85 \\
\hline
\end{tabular}

a MW: molecular weight $(\mathrm{g} / \mathrm{mol})$.

b $C_{\mathrm{s}}$ : water solubility $(\mathrm{mg} / \mathrm{L})$.

c $K_{\text {ow }}$ : octanol-water partition coefficient, taken from Ref. [10].

d $\rho$ : density $\left(\mathrm{g} / \mathrm{cm}^{3}\right)$. Data of $C_{\mathrm{s}}$ and $\rho$ are taken from European Union Risk Assessment Report.

e $V_{\mathrm{s}}$ : molar volume $\left(\mathrm{cm}^{3} / \mathrm{mol}\right)$, calculated from MW and $\rho, V_{\mathrm{s}}=\mathrm{MW} / \rho$.

f MV: molecule volume $\left(\AA^{3}\right)$, calculated according to the Ref. [37].

are supplied in the SI. All CNTs were characterized for their surface oxygen and carbon atomic percentage using X-ray photoelectron spectroscopy (XPS), and specific surface area and pore volume with multipoint Brunauer-Emmett-Teller (BET) measurements. Selected structural properties of pristine and oxidized CNTs are summarized in Table 2.

\subsection{Batch experiments}

Batch equilibrium experiments were conducted to explore the adsorption behavior of OPEs on CNTs. A fixed amount of sorbent from 0.2 to $4 \mathrm{mg}$ was added, depending on the sorption capacity of the CNTs, into an 8-mL Teflon-lined screw cap glass vial. The individual OPE solutions, dissolved in a background solution containing $0.01 \mathrm{~mol} / \mathrm{L} \mathrm{NaCl}$ and $200 \mathrm{mg} / \mathrm{L} \mathrm{NaN}_{3}$ (as bioinhibitor), were prepared in vials with a series of initial concentrations $(0.1-100.0 \mathrm{mg} / \mathrm{L})$. Then the samples were mixed in the dark for $4 \mathrm{~d}$ at $25 \pm 1^{\circ} \mathrm{C}$. The preliminary equilibrium experimental results indicated that equilibration time was less than $4 \mathrm{~d}$. The samples were then centrifuged $(4000 \mathrm{rpm}, 30 \mathrm{~min}$ ) and the concentrations of sorbates in the supernatants were determined by high-performance liquid chromatography-mass spectrometry (Shimadzu, LC-MS 2020). During the sorption process, the $\mathrm{pH}$ remained unchanged at neutral since all the OPEs are nonionic. The results of duplicate samples were averaged and reported. Due to negligible mass losses $(<6 \%)$ as indicated in control tests without sorbents, the amounts of adsorbed OPEs were calculated by mass difference.

\subsection{Sorption models and data analysis}

Three nonlinear isotherm models, The Dubinin-Ashtakhov model (DAM), Langmuir model (LM), and Freundlich model (FM), were used to describe the sorption isotherms. These models have been successfully used for CNTs, activated carbon, and geosorbents [20]. The details of the modeling and data analysis are shown in the SI.

\subsection{Spectroscopic measurements}

ATR-FTIR measurements were performed using a ThermoNicolet Nexus 6700 FTIR spectrometer equipped with a horizontal attenuated total reflectance (HATR) cell (PIKE Tech) and a liquidnitrogen-cooled mercury-cadmium-telluride (MCT) detector. The infrared spectra were collected using 512 scans per spectrum at a resolution of $4 \mathrm{~cm}^{-1}$. The spectra of OPE reference standards were obtained by subtracting the spectrum of pure acetonitrile from the spectra of $1000 \mathrm{mg} / \mathrm{L}$ OPE solutions in acetonitrile. The IR spectra of sorption samples were obtained by mixing freeze-dried sorption samples ( $2 \mathrm{mg}$ ) with $\mathrm{KBr}(98 \mathrm{mg}$ ) to make a pellet, and analyzing 
Table 2

Selected structural properties of CNTs and their purity.

\begin{tabular}{|c|c|c|c|c|c|c|c|c|}
\hline \multirow[t]{2}{*}{ CNTs } & \multirow[t]{2}{*}{ Outer DI $(\mathrm{nm})^{\mathrm{a}}$} & \multirow[t]{2}{*}{ Length $(\mathrm{nm})^{\mathrm{a}}$} & \multirow[t]{2}{*}{ Purity (\%) ${ }^{\mathrm{a}}$} & \multicolumn{2}{|c|}{ Surface atom $(\%)^{\mathrm{b}}$} & \multirow[t]{2}{*}{$A_{\text {surf }}{ }^{\mathrm{c}}\left(\mathrm{m}^{2} / \mathrm{g}\right)$} & \multirow[t]{2}{*}{$V_{\text {meso }}{ }^{c}\left(\mathrm{~cm}^{3} / \mathrm{g}\right)$} & \multirow[t]{2}{*}{$V_{\text {micro }}{ }^{\mathrm{c}}\left(\mathrm{cm}^{3} / \mathrm{g}\right)$} \\
\hline & & & & $\mathrm{C}$ & $\mathrm{O}$ & & & \\
\hline MWCNT & $20-40$ & $5-15$ & $>95$ & 97.0 & 3.0 & 89 & 0.425 & 0.071 \\
\hline O-MWCNT & $20-40$ & $5-15$ & $>95$ & 92.4 & 7.6 & 114 & 0.582 & 0.106 \\
\hline SWCNT & $\leq 2$ & $5-15$ & $>95$ & 98.6 & 1.4 & 460 & 0.887 & 0.195 \\
\hline O-SWCNT & $\leq 2$ & $5-15$ & $>95$ & 96.2 & 3.8 & 472 & 0.904 & 0.199 \\
\hline
\end{tabular}

a Provided by the supplier.

b Surface carbon and oxygen content were analyzed by X-ray photoelectron spectroscopy (XPS).

c $A_{\text {surf }}$ (surface area), $V_{\text {meso }}$ (mesopore volume), $V_{\text {micro }}$ (micropore volume) were calculated from the adsorption-desorption isotherms of $\mathrm{N}_{2}$ at $77 \mathrm{~K}$ by multipoint $\mathrm{BET}$, BJH, and DR methods, respectively.

with ATR-FTIR. Raman analysis was conducted with a Renishaw inVia Raman microscope with $1 \mathrm{~cm}^{-1}$ resolution at $532 \mathrm{~nm}$ excitation energy. The laser power was maintained at $1 \mathrm{~mW}$ throughout the experiment and the acquisition time was $5 \mathrm{~s}$.

\section{Results and discussion}

\subsection{Characterization of CNTs}

The oxygen contents on the CNT surfaces as characterized by XPS increased from $3.0 \%$ to $7.6 \%$ for MWCNTs and $1.4 \%$ to $3.8 \%$ for SWCNTs after $\mathrm{HNO}_{3}$ oxidation treatment (Table 2). The surface oxygen-containing groups were mainly carbonyls, hydroxyls, esters, and carboxyls (Figs. S2 and S3). The BET analysis showed that the oxidation induced a slightly higher surface area and pore volume for the CNTs (Table 2). Though the TEM analysis revealed that the morphology of CNTs had undergone no significant changes upon $\mathrm{HNO}_{3}$ oxidation (Fig. S4), the increased intensity ratio of the disorder-induced D-band to the tangential mode G-band, $I_{\mathrm{D}} / I_{\mathrm{G}}$, confirmed that the oxidation resulted in sidewall damage on CNTs, which caused an increase in structural disorder (Fig. S5).

\subsection{Sorption Isotherms}

The isotherms of the six OPEs on the four CNTs were nonlinear (Fig. 1), implying the presence of heterogeneous sorption sites [21]. Three nonlinear sorption models, DAM, LM, and FM, were used to fit the isotherms, and the results are listed in Table 3 and SI Tables S1
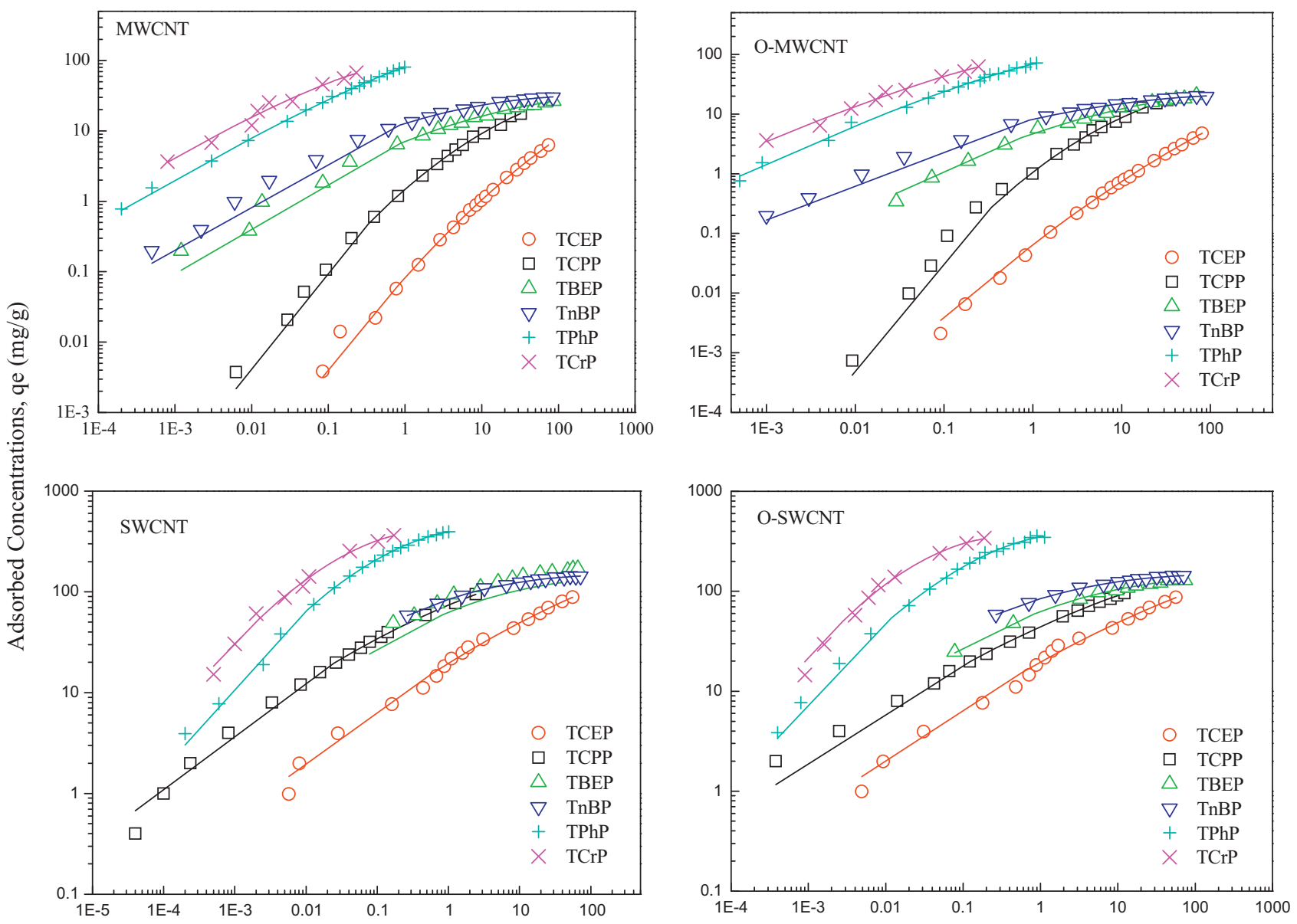

Equilibrium concentration, $\mathrm{Ce}(\mathrm{mg} / \mathrm{L})$

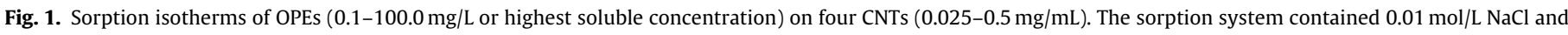
$200 \mathrm{mg} / \mathrm{L} \mathrm{NaN}_{3}$ at $25 \pm 1{ }^{\circ} \mathrm{C}$. Solid lines represent the DAM model results. 
Table 3

Calculated results and goodness of fit for adsorption of OPEs on CNTs based on DAM.

\begin{tabular}{|c|c|c|c|c|c|c|c|c|c|c|c|}
\hline OPEs & Adsorbent & $Q^{0}(\mathrm{mg} / \mathrm{g})$ & $E(\mathrm{~kJ} / \mathrm{mol})$ & $b$ & $r_{\text {adj }}^{2}$ & MWSE & $Q_{m}^{b}(\mathrm{mg} / \mathrm{g})$ & $Q^{0} / Q_{\mathrm{m}}$ & Reduction percentage $^{c}$ & $V_{\text {sorption }}\left(\mathrm{mm}^{3} / \mathrm{g}\right)$ & Kat $C_{\mathrm{e}}=0.01 C_{\mathrm{s}}{ }^{\mathrm{d}}$ \\
\hline \multirow[t]{4}{*}{ TCEP } & MWCNT & $57.2 \pm 6.6^{\mathrm{e}}$ & $11.8 \pm 0.4$ & $1.66 \pm 0.07$ & 0.995 & 0.025 & 72.5 & 0.789 & \multirow[t]{2}{*}{$8.22 \%$} & 40.31 & $0.08 \pm 0.02$ \\
\hline & O-MWCNT & $52.5 \pm 5.0$ & $11.0 \pm 0.3$ & $1.53 \pm 0.05$ & 0.999 & 0.026 & 92.8 & 0.566 & & 37.00 & $0.06 \pm 0.01$ \\
\hline & SWCNT & $237.6 \pm 46$ & $21.1 \pm 1.2$ & $1.56 \pm 0.19$ & 0.996 & 0.027 & 374.7 & 0.634 & \multirow[t]{2}{*}{$5.51 \%$} & 167.44 & $1.26 \pm 0.25$ \\
\hline & O-SWCNT & $224.5 \pm 55$ & $21.4 \pm 1.5$ & $1.57 \pm 0.25$ & 0.994 & 0.025 & 384.4 & 0.584 & & 158.21 & $1.22 \pm 0.29$ \\
\hline \multirow[t]{4}{*}{ TCPP } & MWCNT & $41.4 \pm 8.5$ & $14.4 \pm 0.6$ & $1.98 \pm 0.29$ & 0.995 & 0.015 & 71.1 & 0.582 & \multirow[t]{2}{*}{$13.77 \%$} & 32.14 & $0.90 \pm 0.18$ \\
\hline & O-MWCNT & $35.7 \pm 4.0$ & $14.4 \pm 0.3$ & $2.28 \pm 0.20$ & 0.998 & 0.053 & 91.1 & 0.392 & & 27.72 & $0.85 \pm 0.10$ \\
\hline & SWCNT & $224.1 \pm 12$ & $25.7 \pm 0.3$ & $1.85 \pm 0.07$ & 0.996 & 0.014 & 367.7 & 0.609 & \multirow{2}{*}{$11.24 \%$} & 173.99 & $12.42 \pm 0.67$ \\
\hline & O-SWCNT & $198.9 \pm 11$ & $22.5 \pm 0.3$ & $1.63 \pm 0.07$ & 0.993 & 0.017 & 377.3 & 0.527 & & 154.43 & $8.60 \pm 0.47$ \\
\hline \multirow[t]{4}{*}{ TBEP } & MWCNT & $32.8 \pm 0.9$ & $21.8 \pm 0.2$ & $2.00 \pm 0.09$ & 0.997 & 0.031 & 60.9 & 0.537 & \multirow[t]{2}{*}{$20.80 \%$} & 32.06 & $1.34 \pm 0.04$ \\
\hline & O-MWCNT & $25.9 \pm 1.5$ & $20.4 \pm 0.3$ & $2.12 \pm 0.17$ & 0.995 & 0.028 & 78.0 & 0.332 & & 25.39 & $1.02 \pm 0.06$ \\
\hline & SWCNT & $180.6 \pm 2.3$ & $27.0 \pm 0.3$ & $2.73 \pm 0.11$ & 0.996 & 0.005 & 314.8 & 0.574 & \multirow[t]{2}{*}{$21.93 \%$} & 177.06 & $11.15 \pm 0.15$ \\
\hline & O-SWCNT & $141.0 \pm 2.3$ & $26.8 \pm 0.4$ & $2.55 \pm 0.12$ & 0.996 & 0.008 & 323.0 & 0.437 & & 138.24 & $8.35 \pm 0.14$ \\
\hline \multirow[t]{4}{*}{ TnBP } & MWCNT & $32.0 \pm 0.3$ & $22.6 \pm 0.2$ & $2.18 \pm 0.05$ & 0.999 & 0.039 & 55.0 & 0.582 & \multirow[t]{2}{*}{$33.75 \%$} & 32.99 & $47.58 \pm 0.45$ \\
\hline & O-MWCNT & $21.2 \pm 0.2$ & $22.5 \pm 0.1$ & $2.11 \pm 0.03$ & 0.998 & 0.011 & 70.4 & 0.301 & & 21.86 & $30.57 \pm 0.29$ \\
\hline & SWCNT & $202.6 \pm 4.2$ & $29.7 \pm 0.9$ & $1.94 \pm 0.13$ & 0.993 & 0.002 & 284.1 & 0.713 & \multirow[t]{2}{*}{$27.49 \%$} & 208.87 & $353.27 \pm 7.33$ \\
\hline & O-SWCNT & $146.9 \pm 1.2$ & $26.1 \pm 0.5$ & $2.51 \pm 0.01$ & 0.996 & 0.012 & 291.5 & 0.504 & & 151.44 & $275.09 \pm 2.25$ \\
\hline \multirow[t]{4}{*}{ TPhP } & MWCNT & $101.5 \pm 2.1$ & $11.9 \pm 0.1$ & $1.17 \pm 0.03$ & 0.999 & 0.005 & 68.0 & 1.493 & \multirow[t]{2}{*}{$17.93 \%$} & 84.16 & $595.84 \pm 12.33$ \\
\hline & O-MWCNT & $83.3 \pm 2.7$ & $11.8 \pm 0.3$ & $1.24 \pm 0.07$ & 0.996 & 0.008 & 87.1 & 0.956 & & 69.07 & $480.93 \pm 15.59$ \\
\hline & SWCNT & $412.4 \pm 3.2$ & $14.7 \pm 0.1$ & $1.75 \pm 0.03$ & 0.999 & 0.011 & 351.5 & 1.173 & \multirow[t]{2}{*}{$10.38 \%$} & 341.96 & $4944.34 \pm 38.36$ \\
\hline & O-SWCNT & $369.6 \pm 7.0$ & $14.0 \pm 0.3$ & $1.74 \pm 0.09$ & 0.996 & 0.005 & 360.6 & 1.025 & & 306.47 & $3871.04 \pm 73.31$ \\
\hline \multirow[t]{4}{*}{ TcrP } & MWCNT & $74.1 \pm 6.6$ & $12.2 \pm 0.8$ & $1.24 \pm 0.22$ & 0.981 & 0.031 & 69.0 & 1.074 & \multirow[t]{2}{*}{$7.29 \%$} & 63.88 & $2469.31 \pm 219.9$ \\
\hline & O-MWCNT & $68.7 \pm 3.7$ & $11.8 \pm 0.5$ & $1.20 \pm 0.13$ & 0.992 & 0.014 & 88.4 & 0.777 & & 59.22 & $2087.26 \pm 112.4$ \\
\hline & SWCNT & $388.9 \pm 13$ & $13.8 \pm 0.3$ & $1.71 \pm 0.13$ & 0.995 & 0.011 & 356.7 & 1.090 & \multirow[t]{2}{*}{$9.82 \%$} & 335.26 & $20441.21 \pm 683.3$ \\
\hline & O-SWCNT & $350.7 \pm 9.8$ & $13.2 \pm 0.3$ & $1.88 \pm 0.13$ & 0.996 & 0.028 & 366.0 & 0.958 & & 302.33 & $16885.87 \pm 471.9$ \\
\hline
\end{tabular}

a The meaning of fitting parameters, including $Q^{0}, E, b$, for DAM, and coefficients for regression analysis, including $r_{\text {adj }}^{2}$, MWSE, are detailed in SI.

b $Q_{m}$ is monolayer sorption capacity ( $\mathrm{mg} / \mathrm{g}$ ), calculated by the method described in Ref. [22] (detailed in SI).

c Reduction percentage means percent reduction of surface coverage when $\mathrm{CNTs}$ were compared to oxidized CNTs, equal to $\left[\left(Q^{0} / Q_{\mathrm{m}}\right)_{\mathrm{CNT}}-\left(Q^{0} / Q_{\mathrm{m}}\right)_{\mathrm{O}-\mathrm{CNT}}\right] /\left(Q^{0} / Q_{\mathrm{m}}\right)_{\mathrm{CNT}} \times 100 \%$,

${ }^{d} K$ is single point sorption coefficient $(\mathrm{L} / \mathrm{g})$, calculated at $C_{\mathrm{e}}=0.01 C_{\mathrm{s}}$ based on the fitting results of DAM.

e Mean \pm standard deviation.

and S2, respectively. The fitting performance was evaluated by the adjusted $r^{2}\left(r_{\text {adj }}^{2}\right)$ and mean weighted square errors (MWSE), which are detailed in the SI. The results showed that DAM could describe the experimental isotherms well over a four order of magnitude concentration range (Fig. 1, $r_{\text {adj }}^{2}>0.981$, MWSE < 0.053).

In comparison, low $r_{\text {adj }}^{2}$ and high MWSE values were obtained when LM and FM were applied (Tables S1 and S2). Though FM (Fig. S6) and LM (Fig. S7) were suitable in the high- and middleconcentration range, they failed to describe the sorption behaviors at low concentrations for all isotherms. LM, usually applicable to describe monolayer adsorption on homogeneous surfaces, is unsuitable for CNTs because the presence of high-energy sorption sites such as defects, functional groups, and interstitial or groove regions between CNT bundles [22] makes the CNTs' surfaces heterogeneous. FM, an empirical equation, failed to describe the sorption behavior of organic pollutants on CNTs over a wide concentration range [23].

\subsection{Influence of OPE Properties on Sorption}

The sorption affinity of OPEs increased in the order $\mathrm{TCEP}<\mathrm{TCPP}<\mathrm{TBEP}<\mathrm{TnBP}<\mathrm{TPhP}<\mathrm{TCrP}$ (Fig. 1). This affinity sequence could be attributed to the hydrophobicity of the OPEs (as measured by $\log K_{\text {ow }}$, Table 1 ), suggesting that the hydrophobic interaction derived from the van der Waals forces dominated the sorption of OPEs on CNTs. The sequence was also supported by the single point sorption coefficient, $K$ (at $C_{\mathrm{e}}=0.01 C_{\mathrm{s}}$, Table 3 ), except that the sorption of TBEP on SWCNTs $(K=11.15)$ and O-SWCNTs (8.35) was less than that of TCPP. The reversed order of TBEP and TCPP could be attributed to the fact that the more hydrophobic TBEP compared to TCPP ( $\log K_{\text {ow }} 3.75$ vs. 2.59$)$ has higher water solubility (1300 mg/L vs. $1080 \mathrm{mg} / \mathrm{L})$.
The normalization of the sorption coefficient by $K_{\mathrm{ow}}$, which screened out the hydrophobic effect [21], showed that $K / K_{\text {ow }}$ followed the order TBEP $<\mathrm{TnBP}<\mathrm{TCPP}<\mathrm{TCEP}<\mathrm{TPhP}<\mathrm{TCrP}$ (Fig. S8). The $K / K_{\text {ow }}$ values of OPEs with aromatic substituents (i.e. TPhP, TCrP) were significantly higher than those of aliphatic OPEs (i.e. TBEP, TnBP, TCPP, TCEP), highlighting the importance of $\pi-\pi$ EDA interaction in the sorption of aromatic OPEs on CNTs.

Our results demonstrated that the morphology of OPE molecules had insignificant impact on their sorption. The molecular morphology of sorbates is reported to determine the availability of different sorption sites on CNTs [24]. For example, the benzene ring of naphthol can match the hexagonal carbon rings on the CNT surface, whereas 2-phenyphenol must bind to the tube surface with one separate aromatic ring offsetting the carbon rings of CNTs, which consequently reduces the contact area and thus the sorption coefficient [25]. In our study, the influence of different molecular configurations between TPhP with benzene and TCrP with methylbenzene was negligible since they had comparable $K / K_{\mathrm{ow}}$ values (15.3 L/kg vs. $19.2 \mathrm{~L} / \mathrm{kg}$, Fig. S8). The influence of the OPEs' shape on their sorption affinity was not strong because OPE molecules, with their three-dimensional structures, could not contact with the CNT walls as closely as the substituent molecules (e.g. benzene, methylbenzene) (Fig. S1).

Distinct influence of the molecular size of organic compounds on sorption has been observed. Wang et al. [21] reported that the volume sorption capacity ( $\left.V_{\text {sorption }}\right)$ of atrazine, phenanthrene, and lindane was negatively correlated with their molecular sizes due to the steric hindrance of micropores on the access of these large molecules to the sorption sites. However, this molecular sieving effect was not apparent in the present study since $V_{\text {sorption }}$, calculated from the mass capacity divided by the solid phase density, did not relate to the molecular size (Table 3 ). The possible reason accounting for the different results was that the range of OPE molecular sizes $\left(194.85-367.77 \AA^{3}\right)$ in this study is relative smaller 
than that in Wang's study (33.4-241.9 $\AA^{3}$ ). Therefore, the molecular sieving effect of CNT pore size was not distinct in our study.

\subsection{Influence of CNT surface properties on sorption}

The maximum sorption capacities as defined by DAM $\left(Q^{0}, \mathrm{mg} / \mathrm{g}\right)$ followed the order O-MWCNT $<$ MWCNT $<$ O-SWCNT $<$ SWCNT (Table 3). $Q^{0}$ for SWCNT was 4-6 times higher than that for MWCNT, which was comparable to their surface area ratio $(460 / 89=5.17)$. The poor correlation between $Q^{0}$ and hydrophobicity of OPEs indicated that $Q^{0}$ was independent of sorption affinity (Fig. S9).

The maximum surface coverage, calculated by dividing $Q^{0}$ by the monolayer sorption capacity $\left(Q_{\mathrm{m}}\right)$, was in the range $30-79 \%$ for aliphatic OPEs (Table 3). The relatively low surface coverage was mainly caused by the assumption that the CNT surfaces available for $\mathrm{N}_{2}$ molecules in the BET method were completely available for OPEs, which thus overestimated the $Q_{m}$ value [17].

The low surface coverage also implied that aliphatic OPEs could not occupy all available sorption sites on CNTs. The oxygencontaining functional groups on CNTs could readily combine with water molecules by $\mathrm{H}$ bonding, which made them inaccessible for hydrophobic compounds [24]. This explanation is in accordance with the reduced surface coverage observed after the oxidation of CNTs (Table 3). Furthermore, the reduction in coverage percentage for highly hydrophobic OPEs (e.g., 33.75\% and $27.49 \%$ of TnBP) was greater than for those with low hydrophobicity (e.g. $8.22 \%$ and $5.51 \%$ of TCEP, Fig. 2, Table 3), indicating that the surface oxidation of CNTs had more a significant influence on hydrophobic OPEs than on hydrophilic OPEs.

For aromatic OPEs (TPhP, TCrP), the maximum surface coverage, $Q^{0} / Q_{m}$, was in the range $78-149 \%$ (Table 3 ). Three reasons could possibly account for the appreciably higher surface coverage of aromatic OPEs compared with that of aliphatic ones. First, the $\pi-\pi$ bond interaction between the aromatic moiety of OPEs and the Cring of CNTs could disrupt the van der Waals force between the CNT bundles and consequently increase their effective surface area by preventing them from aggregating [24]. Second, the dissociated species of oxygen-containing functional groups (i.e. $-\mathrm{COO}^{-},-\mathrm{O}^{-}$) on the CNT surface, which predominate in natural waters $\left(\mathrm{pH}_{\mathrm{pzc}}\right.$ of the CNTs $\approx 4.3$, measured in the lab), could serve as strong ndonors in the $n-\pi$ EDA interaction with aromatic OPEs, thereby enhancing these OPEs' sorption affinity and capacity on the oxidized surface of CNTs. Third, self-stacking interactions could occur

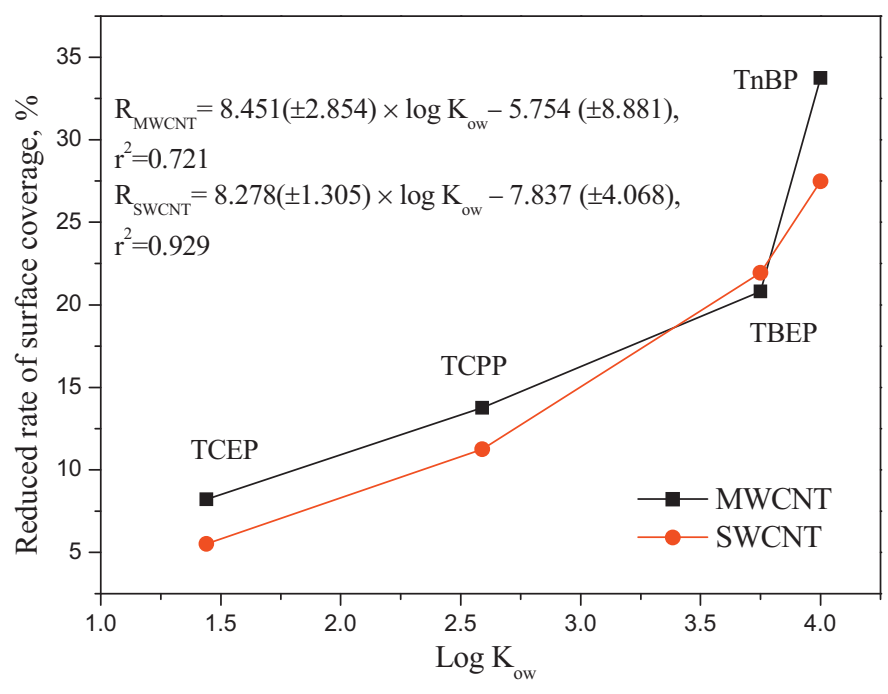

Fig. 2. Correlation of reduced rate of surface area coverage with $\log K_{\text {ow }}$ values of aliphatic OPEs.

between the aromatic domains of free and adsorbed OPE molecules, and a multilayer of sorbates may form on the CNT surface [26].

\subsection{Raman analysis}

Raman spectroscopy is a powerful tool to characterize the structure of $\mathrm{sp}^{2}$ carbon nanostructures due to its high sensitivity [27]. Because SWCNTs have a similar surface structure to that of MWCNTs but an elevated sorption capacity, only SWCNTs were selected for spectroscopic analysis.

Three phonon modes were observed for pristine and OPEadsorbed SWCNTs (Fig. 3), which are the radial breathing mode (RBM) in the range $100-300 \mathrm{~cm}^{-1}$, the disorder-induced D-band at $1342 \mathrm{~cm}^{-1}$, and the tangential mode $\mathrm{G}$-band at $1586 \mathrm{~cm}^{-1}$. Because sorption had no impact on the defects of CNTs and therefore on the D-band, as evidenced by the following FTIR analysis, the Raman analysis was focused on the RBM and G-band. For comparison purposes, the intensities of all spectra in Fig. 3 were normalized to yield the same intensity for the G-band.

RBM reflects the in-phase vibration of carbon atoms in a direction perpendicular to the tube axis [28]. As shown in Fig. 3a, a
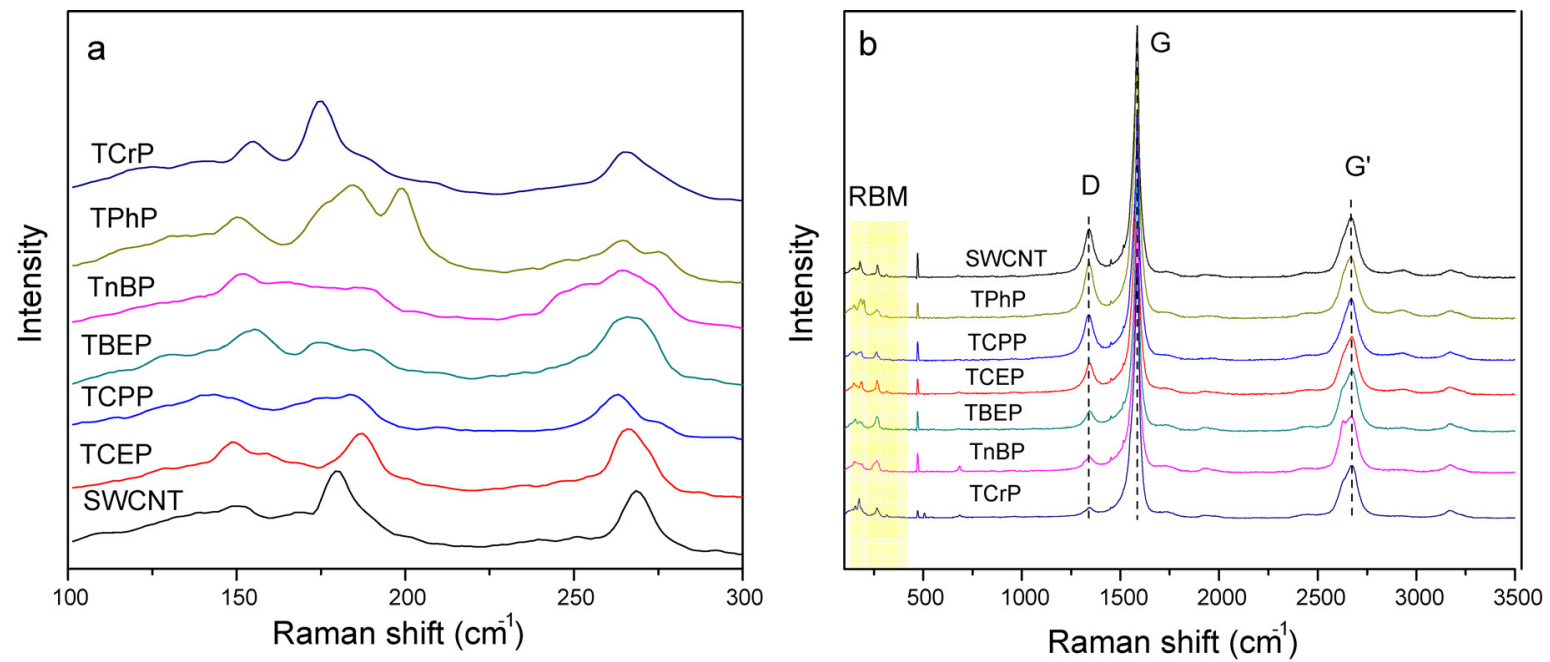

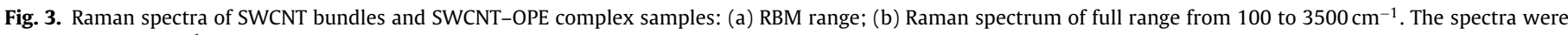
collected with $1 \mathrm{~cm}^{-1}$ resolution at $532 \mathrm{~nm}$ excitation energy. 

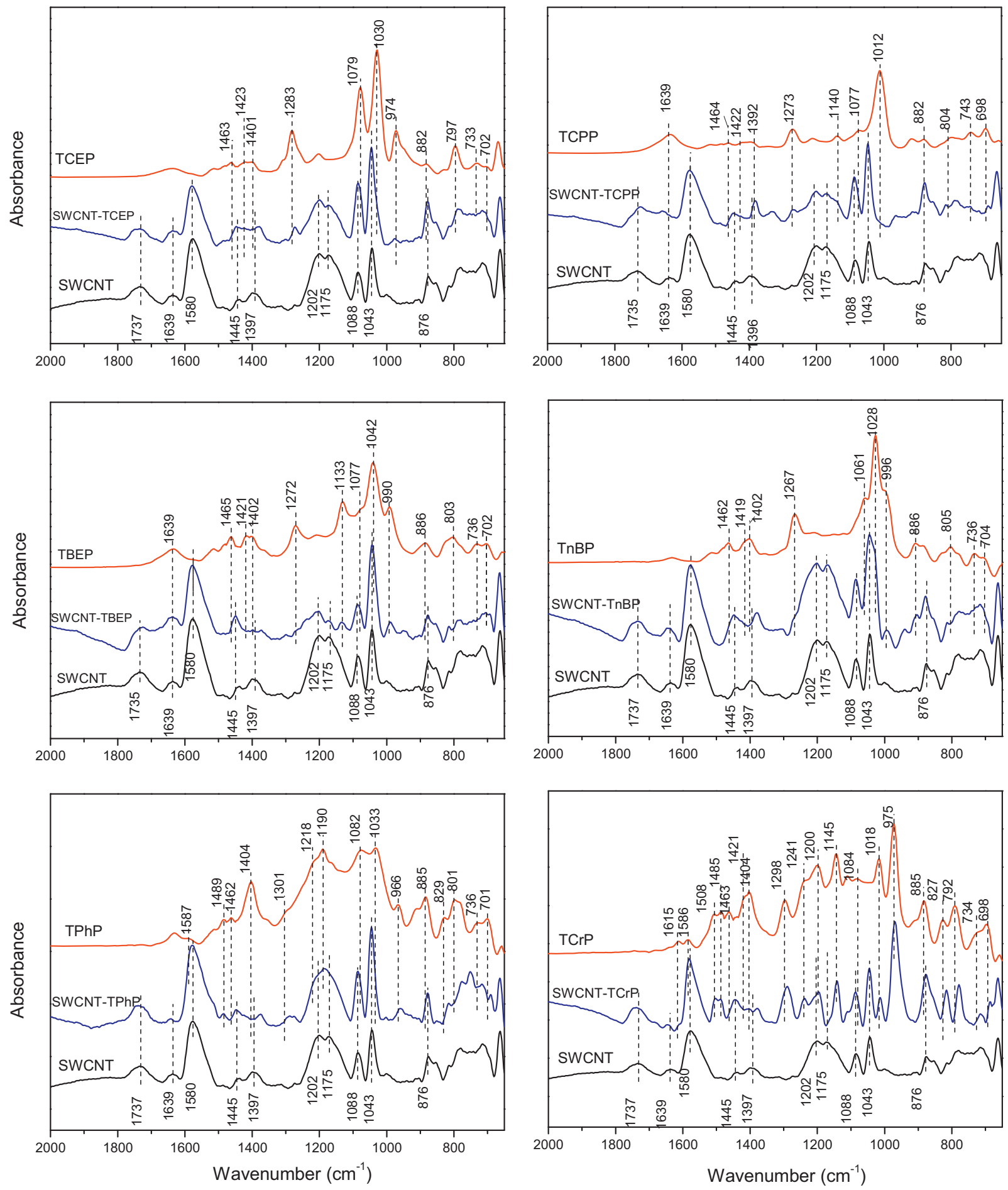

Fig. 4. FTIR spectra of SWCNT bundles, OPEs, and SWCNT-OPE complex samples. The spectra were collected using 512 scans per spectrum at a resolution of $4 \mathrm{~cm}^{-1}$.

weak peak at $150 \mathrm{~cm}^{-1}$ and strong peaks at 179 and $268 \mathrm{~cm}^{-1}$ were resolved, corresponding to nanotubes of $1.67 \mathrm{~nm}, 1.38 \mathrm{~nm}$, and $0.91 \mathrm{~nm}$ in diameter, respectively, according to the relation $\omega_{\mathrm{RBM}}\left(\mathrm{cm}^{-1}\right)=234 / d+10$, where the $d$ is the diameter of a nanotube [29]. The peak position and shape changed significantly upon OPE sorption, suggesting that the OPEs sorbed on the external walls of SWCNTs affect the strain of nanotubes in the radial direction.

The changes in RBM peaks may be attributed to the $\pi-\pi$ interaction and van der Waals forces. The $\pi-\pi$ interaction between the aromatic moiety of OPEs and SWCNT sidewalls leads to the "mode hardening effect" [30], which constrains the radial vibration of SWCNTs and consequently results in a high frequency shift of the RBM. In addition, this mode hardening effect may be overwhelmed by the van der Waals interactions between OPEs and SWCNTs. It has been reported that the van der Waals forces between nanotubes could lead to substantial deformation of the nanotubes themselves [31]. In addition, the radial deformation of CNT bundles induced by nanotube-substrate van der Waals forces has also been reported [28]. In the current study, the sorption of OPEs could interfere with the van der Waals forces between the nanotubes, which causes debundling [32], and the OPEs-SWCNTs van der Waals interaction could lead to a new radial deformation of the nanotubes. This 
hypothesis is in agreement with our experimental observation that the suspensions of SWCNTs adsorbed with OPEs were stable for $5 \mathrm{~d}$ while that of pristine SWCNTs aggregated in $24 \mathrm{~h}$. Because of the different strength of van der Waals forces between nanotube bundles and SWCNTs with various OPEs, no monotonic RBM shift was observed.

The G-band, associated with carbon atom vibrations in the tangential direction, is a Raman characteristic region that is sensitive to charge-transfer effects [33]. As shown in Fig. 3b, no significant shift of G-bands was observed. This result indicated that compared with the covalent interaction, the $\pi-\pi$ EDA interaction between aromatic OPEs and the C-rings of SWCNTs is too weak to be detectable by Raman scattering. In line with our observations, Fagan et al. found that charge transfer from 1,2-dichlorobenzene (DCB) to SWCNTs is negligibly small (only 0.004 electron/DCB-molecule) [34]. Polar interactions may also generate a charge transfer effect [35], but such a scenario cannot occur in our experiments since all six OPEs lack polar or ionic substituent groups.

\subsection{ATR-FTIR study}

As an important complementary probe to Raman scattering, ATR-FTIR spectroscopy was used to investigate the molecular interactions between the SWCNTs and OPEs. Fig. 4 shows the IR spectra of OPEs, SWCNT, and OPE-adsorbed SWCNT samples. The detailed IR assignments are supplied in SI Table S3 and Fig. S10.

In general, most IR bands of OPEs did not shift significantly upon sorption, indicating that van der Waals forces between OPEs and SWCNT bundles, the main driving force of the sorption, could not result in pronounced IR peak shifts (Fig. 4). Instead, other interactions were observed. First, $v(\mathrm{P}=\mathrm{O})$ bands of TCEP downshifted from $1283 \mathrm{~cm}^{-1}$ to $1273 \mathrm{~cm}^{-1}$ after sorption, indicating that the sorption of TCEP on SWCNTs involves the $\mathrm{P}=\mathrm{O}$ band. As reported by Mäkie et al. [36], a great downshift of the $v(\mathrm{P}=0)$ band by $45-55 \mathrm{~cm}^{-1}$ was observed when trimethylphosphate (TMP), an OPE with a structure similar to TCEP, was adsorbed to Fe oxides and $\sim 20 \mathrm{~cm}^{-1}$ when adsorbed to Fe hydroxide. The large downshift was attributed to the interactions with Fe Lewis acid sites $(-\mathrm{P}=\mathrm{O}-\mathrm{Fe})$, and the relatively small one to hydrogen bonding $(-\mathrm{P}=\mathrm{O} \cdots \mathrm{H}-)$. In our case, the downshift of only $10 \mathrm{~cm}^{-1}$ for $v(\mathrm{P}=\mathrm{O})$ bands implied that hydrogen bonding is more likely to occur between the $\mathrm{P}=\mathrm{O}$ oxygen in TCEP, acting as a Brønsted base, and the SWCNT surface OH groups, acting as a Brønsted acid [36]. Except for TCEP, $v(\mathrm{P}=0)$ in the other OPEs did not shift because the relatively large substituent groups of these OPEs inhibit the $\mathrm{P}=\mathrm{O}$ oxygen from being accessible to SWCNT surface $\mathrm{OH}$ groups.

In addition, Fig. 4 shows that all the $\delta\left(\mathrm{CH}_{3}\right) / \delta\left(\mathrm{CH}_{2}\right)$ bands of OPEs at $1392-1423 \mathrm{~cm}^{-1}$ (except TPhP) downshifted by $11-27 \mathrm{~cm}^{-1}$ upon sorption. In contrast, most $v(\mathrm{P}-\mathrm{O}-\mathrm{C})$ bands at $1018-1030 \mathrm{~cm}^{-1}$ and $882-886 \mathrm{~cm}^{-1}$ did not shift. The results indicated that hydrogen bonding occurred between the $\mathrm{H}$ atom in the substituent group (R), rather than the $\mathrm{O}$ atom in-P-O-(R) of the OPEs, and with the SWCNT surface OH groups.

The $\pi-\pi$ EDA interaction is an important non-covalent interaction. For TPhP and TCrP, the stretching modes of benzene rings, such as 1587 and $1489 \mathrm{~cm}^{-1}$ for TPhP, 1508 and $1485 \mathrm{~cm}^{-1}$ for TCrP, did not change (Fig. 4 and Table S4). However, the in-plane deformation vibration $\delta(\mathrm{CH})$ bands of these two OPEs at $1301 \mathrm{~cm}^{-1}$ and $1298 \mathrm{~cm}^{-1}$ downshifted to $1290 \mathrm{~cm}^{-1}$ and $1289 \mathrm{~cm}^{-1}$, respectively; The out-of-plane deformation vibration $\gamma(\mathrm{CH})$ bands at 966 , $829 \mathrm{~cm}^{-1}$ for TPhP, and $975,827 \mathrm{~cm}^{-1}$ for TCrP also downshifted to $959,815 \mathrm{~cm}^{-1}$, and $970,816 \mathrm{~cm}^{-1}$, respectively. Consistent with the our Raman analysis, the IR spectra clearly prove the existence of $\pi-\pi$ EDA interactions between aromatic moieties of the OPEs and SWCNT sidewalls, which restricts the vibration of the
$\mathrm{C}-\mathrm{H}$ band in the direction perpendicular to the benzene ring plane.

\section{Conclusions}

Both OPEs and CNTs inevitably enter into the environment due to their increasingly wide industrial applications. The results of this work show that OPEs can be readily adsorbed on CNTs by multiple mechanisms, including van der Waals interactions, $\pi-\pi$ EDA interactions, and hydrogen bonding. The sorption capacity of OPEs largely depends on the surface area and oxygen-containing functional groups of CNTs. The strong affinity of OPEs toward CNTs not only inhibits the migration of OPEs and therefore reduces their exposure potential, but also adds additional toxicity to CNTs when these CNTs are bio-concentrated in organisms [22]. Therefore, the potential risk of coexistent CNTs and OPEs or other pollutants in the environment should be critically considered. In the present study, the macroscopic sorption behavior of OPEs on CNTs and microscopic OPE-CNT molecular interaction was systematically studied. However, some substances in the environment such as natural organic matters (NOMs) can seriously affect the dispersion and transport of CNTs and subsequently change their sorption properties for OPEs. Therefore, the influence of these environmental components on the fate and transport of OPE-CNT agglomerates merits further investigation.

\section{Acknowledgments}

We gratefully acknowledge support from the National Key Basic Research Program of China (2014CB114402) and National Natural Science Foundation of China $(21007080,41023005)$. We thank Prof. Jing-Fu Liu (RCEES) for the use of Raman experimental facilities and Ms. Feng Ji (Shimadzu (China) Co., Ltd. Beijing Branch) for help with LC-MS analysis.

\section{Appendix A. Supplementary data}

Supplementary data associated with this article can be found, in the online version, at http://dx.doi.org/10.1016/ j.jhazmat.2014.03.030.

\section{References}

[1] A.M. Sundkvist, U. Olofsson, P. Haglund, Organophosphorus flame retardants and plasticizers in marine and fresh water biota and in human milk, J. Environ. Monit. 12 (2010) 943-951.

[2] I. van der Veen, J. de Boer, Phosphorus flame retardants: properties, production, environmental occurrence, toxicity and analysis, Chemosphere 88 (2012) 1119-1153.

[3] I. Mihajlovic, M.V. Miloradov, E. Fries, Application of twisselmann extraction, SPME, and GC-MS to assess input sources for organophosphate esters into soil, Environ. Sci. Technol. 45 (2011) 2264-2269.

[4] X. Wang, J. Liu, Y. Yin, Development of an ultra-high-performance liquid chromatography-tandem mass spectrometry method for high throughput determination of organophosphorus flame retardants in environmental water, J. Chromatogr. A 1218 (2011) 6705-6711.

[5] H.M. Stapleton, S. Klosterhaus, S. Eagle, J. Fuh, J.D. Meeker, A. Blum, T.F. Webster, Detection of organophosphate flame retardants in furniture foam and US house dust, Environ. Sci. Technol. 43 (2009) 7490-7495.

[6] N. Ratola, A. Cincinelli, A. Alves, A. Katsoyiannis, Occurrence of organic microcontaminants in the wastewater treatment process. A mini review, J. Hazard. Mater. 239-240 (2012) 1-18.

[7] P. Westerhoff, Y. Yoon, S. Snyder, E. Wert, Fate of endocrine-disruptor, pharmaceutical, and personal care product chemicals during simulated drinking water treatment processes, Environ. Sci. Technol. 39 (2005) 6649-6663.

[8] E. Fries, I. Mihajlovic, Pollution of soils with organophosphorus flame retardants and plasticizers, J. Environ. Monit. 13 (2011) 2692-2694.

[9] M. Garcia-Lopez, I. Rodriguez, R. Cela, K.K. Kroening, J.A. Caruso, Determination of organophosphate flame retardants and plasticizers in sediment samples using microwave-assisted extraction and gas chromatography with inductively coupled plasma mass spectrometry, Talanta 79 (2009) 824-829. 
[10] T. Reemtsma, J.B. Quintana, R. Rodil, M. Garcia-Lopez, I. Rodriguez, Organophosphorus flame retardants and plasticizers in water and air I. Occurrence and fate, Trac: Trends Anal. Chem. 27 (2008) 727-737.

[11] E.J. Petersen, L. Zhang, N.T. Mattison, D.M. O'Carroll, A.J. Whelton, N. Uddin, T. Nguyen, Q. Huang, T.B. Henry, R.D. Holbrook, K.L. Chen, Potential release pathways, environmental fate, and ecological risks of carbon nanotubes, Environ. Sci. Technol. 45 (2011) 9837-9856.

[12] M. Seifi, D.K. Ross, D.J. Riley, I. Morrison, The dependence of the hydrogen sorption capacity of singlewalled carbon nanotubes on the concentration of catalyst, Carbon 47 (2009) 3184-3191.

[13] H. Mojtaba, B. Nader, Y. Habibollah, Adsorption of mercury ions from synthetic and real wastewater aqueous solution by functionalized multi-walled carbon nanotube with both amino and thiolated groups, Chem. Eng. J. 237 (2014) 217-228.

[14] J.R. Ellerie, O.G. Apul, T. Karanfil, D.A. Ladner, Comparing graphene, carbon nanotubes, and superfine powdered activated carbon as adsorptive coating materials for microfiltration membranes, J. Hazard. Mater. 261 (2013) 91-98.

[15] X. Qu, P.J.J. Alvarez, Q. Li, Applications of nanotechnology in water and wastewater treatment, Water Res. 47 (2013) 3931-3946.

[16] X. Li, H. Zhao, X. Quan, S. Chen, Y. Zhang, H. Yu, Adsorption of ionizable organic contaminants on multi-walled carbon nanotubes with different oxygen contents, J. Hazard. Mater. 186 (2011) 407-415.

[17] F. Wang, J. Yao, K. Sun, B. Xing, Adsorption of dialkyl phthalate esters on carbon nanotubes, Environ. Sci. Technol. 44 (2010) 6985-6991.

[18] C.Y. Lu, H.S. Chiu, Adsorption of zinc(II) from water with purified carbon nanotubes, Chem. Eng. Sci. 61 (2006) 1138-1145.

[19] H.H. Cho, H. Huang, K. Schwab, Effects of solution chemistry on the adsorption of ibuprofen and triclosan onto carbon nanotubes, Langmuir 27 (2011) $12960-12967$.

[20] W. Yan, S. Hu, C. Jing, Enrofloxacin sorption on smectite clays: effects of pH, cations, and humic acid, J. Colloid Interface Sci. 372 (2012) 141-147.

[21] X. Wang, Y. Liu, S. Tao, B. Xing, Relative importance of multiple mechanisms in sorption of organic compounds by multiwalled carbon nanotubes, Carbon 48 (2010) 3721-3728.

[22] K. Yang, B. Xing, Adsorption of organic compounds by carbon nanomaterials in aqueous phase: Polanyi theory and its application, Chem. Rev. 110 (2010) 5989-6008.

[23] M. Kah, X. Zhang, M.T.O. Jonker, T. Hofmann, Measuring and modelling adsorption of PAHs to carbon nanotubes over a six order of magnitude wide concentration range, Environ. Sci. Technol. 45 (2011) 6011-6017.
[24] B. Pan, B. Xing, Adsorption mechanisms of organic chemicals on carbon nanotubes, Environ. Sci. Technol. 42 (2008) 9005-9013.

[25] D. Lin, B. Xing, Adsorption of phenolic compounds by carbon nanotubes: Role of aromaticity and substitution of hydroxyl groups, Environ. Sci. Technol. 42 (2008) 7254-7259

[26] M. Keiluweit, M. Kleber, Molecular-level interactions in soils and sediments: the role of aromatic $\pi$-systems, Environ. Sci. Technol. 43 (2009) 3421-3429.

[27] M.S. Dresselhaus, G. Dresselhaus, R. Saito, A. Jorio, Raman spectroscopy of carbon nanotubes, Phys. Rep. Rev. Sec. Phys. Lett. 409 (2005) 47-99.

[28] Y. Zhang, J. Zhang, H. Son, J. Kong, Z. Liu, Substrate-induced raman frequency variation for single-walled carbon nanotubes, J. Am. Chem. Soc. 127 (2005) $17156-17157$.

[29] A. Jorio, M.A. Pimenta, A.G. Souza, R. Saito, G. Dresselhaus, M.S. Dresselhaus, Characterizing carbon nanotube samples with resonance Raman scattering, New J. Phys. 139 (2003) 1-17.

[30] S. Gotovac, H. Honda, Y. Hattori, K. Takahashi, H. Kanoh, K. Kaneko, Effect of nanoscale curvature of single-walled carbon nanotubes on adsorption of polycyclic aromatic hydrocarbons, Nano Lett. 7 (2007) 583-587.

[31] R.S. Ruoff, J. Tersoff, D.C. Lorents, S. Subramoney, B. Chan, Radial deformation of carbon nanotubes by van der waals forces, Nature 364 (1993) 514-516.

[32] T.G. Hedderman, S.M. Keogh, G. Chambers, H.J. Byrne, In-depth study into the interaction of single walled carbon nanotubes with anthracene and pterphenyl, J. Phys. Chem. B 110 (2006) 3895-3901.

[33] A.M. Rao, P.C. Eklund, S. Bandow, A. Thess, R.E. Smalley, Evidence for charge transfer in doped carbon nanotube bundles from Raman scattering, Nature 388 (1997) 257-259.

[34] S.B. Fagan, A.G. Souza, J.O.G. Lima, J. Mendes, O.P. Ferreira, I.O. Mazali, O.L. Alves, M.S. Dresselhaus, 1,2-Dichlorobenzene interacting with carbon nanotubes, Nano Lett. 4 (2004) 1285-1288.

[35] L. Piao, Q. Liu, Y. Li, Interaction of amino acids and single-wall carbon nanotubes, J. Phys. Chem. C 116 (2012) 1724-1731.

[36] P. Makie, G. Westin, P. Persson, L. Osterlund, Adsorption of trimethyl phosphate on maghemite, hematite, and goethite nanoparticles, J. Phys. Chem. A 115 (2011) 8948-8959.

[37] J.P. Hickey, D.R. Passino-Reader, Linear solvation energy relationships: rules of thumb for estimation of variable values, Environ. Sci. Technol. 25 (1991 1753-1760. 